\title{
From command-line bioinformatics to bioGUI
}

\author{
Markus Joppich ${ }^{\text {Corresp., } 1}$, Ralf Zimmer ${ }^{\text {Corresp. } 1}$ \\ ${ }^{1}$ Department of Informatics, Ludwig-Maximilians-Universität München, Munich, Germany \\ Corresponding Authors: Markus Joppich, Ralf Zimmer \\ Email address: joppich@bio.ifi.Imu.de, zimmer@ifi.Imu.de
}

Bioinformatics is a highly interdisciplinary field providing (bioinformatics) applications for scientists from many disciplines. Installing and starting applications on the command-line $(\mathrm{CL})$ is inconvenient and/or inefficient for many scientists. Nonetheless, most methods are implemented with a command-line interface only. Providing a graphical user interface (GUI) for bioinformatics applications is one step towards routinely making CL-only applications available to more scientists and, thus, towards a more effective interdisciplinary work.

With our bioGUI framework we address two main problems of using CL bioinformatics applications: First, many tools work on UNIX-systems only, while many scientists use Microsoft Windows. Second, scientists refrain from using command-line tools which, however, could well support them in their research. With bioGUl install modules and templates, installing and using $\mathrm{CL}$ tools is made possible for most scientists even on Windows, due to bioGUl's support for Windows Subsystem for Linux (WSL). In addition, bioGUI templates can easily be created, making the bioGUI framework highly rewarding for developers. From the bioGUI repository it is possible to download, install and use bioinformatics tools with just a few clicks. 


\section{From command-line bioinformatics to bioGUI}

${ }_{3}$ Markus Joppich ${ }^{1}$ and Ralf Zimmer ${ }^{1}$

${ }_{4}{ }^{1}$ Department of Informatics, Ludwig-Maximilians-Universität München, Munich, Germany

6 Corresponding author:

Markus Joppich ${ }^{1}$

Email address: joppich@bio.ifi.Imu.de

\section{A ABSTRACT}

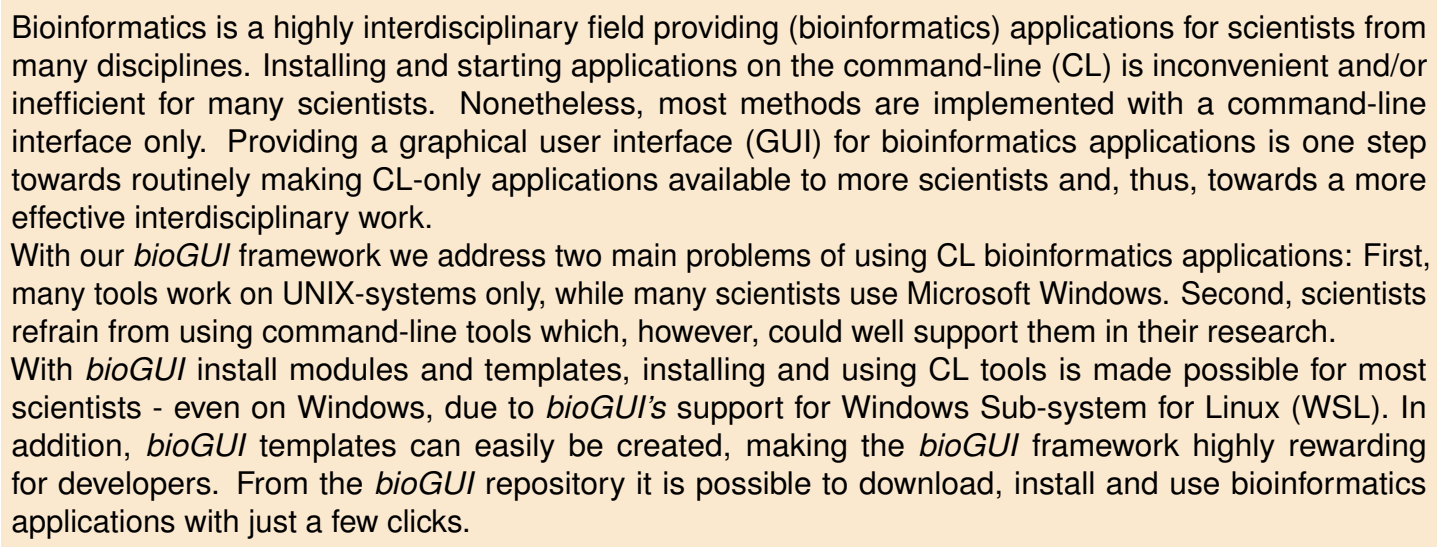

\section{INTRODUCTION}

Many advances in bioinformatics rely on sophisticated applications. Examples are Trinity (Grabherr et al. (2011)) for de novo assembly in conjunction with Trimmomatic (Bolger et al. (2014)), or the HISAT2, StringTie and Ballgown pipeline for transcript-level expression analysis (Pertea et al., 2016). These tools have in common, that, locally installed, only a command-line interface (CLI) is provided, implying a burden for many non-computer affine users (Morais et al., 2018). Jellyfish (Marçais and Kingsford, 2011), Glimmer (Delcher et al., 2007) and HMMer (http://hmmer.org) natively run only in UNIX-environments and require a sophisticated setup on Windows. In addition, the installation of command-line (CL) tools is a challenge for non-computer specialists e.g. due to package dependency resolution. This problem has been addressed by the AlgoRun package (Hosny et al. (2016)), providing a Docker-based repository of tools. Being a web-based service, it is limited to web-applicable data sizes, or local data must be made available to the Docker container in the cloud. While AlgoRun has the advantage of processing data anywhere, it relies on Docker. Docker may be run either on a local workstation or in the cloud. On a local workstation it can induce incompatibilities with existing software (using Hyper-V on Windows). A cloud-based service may conflict with data privacy guide lines (Schadt (2012)), e.g. with respect to a possible de-anonymization of patient samples (Gymrek et al. (2013)). Using Windows Sub-system for Linux (WSL) is often possible in such a scenario: it is provided as an app from the Microsoft Store.

A frequent argument for not providing a graphical user interface (GUI) is the overhead for developing it and the effort to make it really "user centered". Often GUIs are simply deemed unnecessary by application developers. However, one can be sceptical whether non-computer-affine scientists can efficiently use CLIs in their research. In fact, Albert (2016) notes that "Bioinformatics, unfortunately, has quite the number of methods that represent the disconnect of the Ivory Tower". Pavelin et al. (2012) note that software is often developed without a focus on usability of interfaces (for end-users). While this does 
not imply that any GUI is helpful, we argue that without a GUI, the otherwise highly sophisticated CL applications are not very useful for some scientists. Besides, a GUI is often more convenient and helps to avoid using wrong parameters, especially if a software is not yet routinely used in a lab. Smith (2013) also states that GUI-driven applications make daily work in biology or medical labs easier. Smith remarks that many end-users have a "penchant for point and click", not being able to effectively use CL tools. Still they should have the ability to access and analyse their own data. Many proprietary software solutions address this demand: they allow GUI-based data management, while also being extensible via plug-ins. Smith (2015) points out that one of the biggest advantages of such plugins is to combine the power of peer-reviewed algorithms with a user-friendly GUI. Thus, providing a GUI is an important step towards the applicability of methods by end-users. Visne et al. (2009) present a universal GUI for $R$ aiming to close the gap between $R$ developers and GUI-dependent users with limited $R$ scripting skills. Additionally, web-based workflow systems, like Galaxy (Afgan et al., 2016) or Yabi (Hunter et al., 2012) provide means to easily execute (bioinformatics) applications, but aim at more complex workflows. However, both Galaxy and Yabi are designed to be run and maintained by bioinformaticians for several users and are not meant to run on an single, individual basis, like in small labs. More recently Morais et al. (2018) stated that the accessibility of bioinformatics applications is one of the main challenges of contemporary biology, and that one of the main problems for users is the struggle of using CLIs. While a GUI does not make an application user-friendly per se, it helps to make it more accessible by lowering the burden to use it (Xu et al., 2014; Visne et al., 2009; Anslan et al., 2017; Morais et al., 2018; Větrovský et al., 2018).

In recent Microsoft Windows operating systems the WSL feature can be activated. This feature provides a native, non-virtualized Ubuntu environment on Windows, allowing to run most applications that also run on Ubuntu. This solves the problems of running unix-tools on Windows. Remaining problems for scientists aiming to run bioinformatics applications thus might be the installation and usage of CL applications.

Here, bioGUI, an open-source cross-platform framework for making CL applications more accessible via a GUI, is presented. It uses a XML-based domain-specific language (DSL) for template definition, which lowers the initial effort to create a GUI. bioGUI templates for CL applications can easily be scripted. Combined with install modules they provide an efficient and convenient method to deploy bioinformatics applications on Microsoft Windows (via WSL), Mac OS and Linux. bioGUI also addresses protocol/parameter management by saving filled out templates, enabling easy reproducibility of data analyses (Figure 1).

\section{METHODS}

This section first summarizes existing GUI-based systems, then covers the use-case study we performed and goes into detail of how bioGUI works.

\section{Existing (workflow) systems}

There are several (workflow) systems already available. Most prominent in bioinformatics are the Galaxy server and Yabi. In addition, workflow specification languages such as Common Workflow Language (CWL) or Nextflow exist. These workflows do not directly compare to bioGUI because they (usually) require a server infrastructure and are not aimed to run on a local computer. However, they have in common that no CLI is needed to run (bioinformatics) applications.

With RGG a general GUI framework for R already exists. Recently, specialised GUI frameworks, like SEED 2 (Větrovský et al., 2018) or RNA CoMPASS (Xu et al., 2014), have been presented.

Galaxy \& Yabi The Galaxy server is a well known workflow system in bioinformatics (Afgan et al., 2016). While bioGUI does not aim to be a workflow system like Galaxy, e.g. allowing data management, there are similarities. For instance, Galaxy also provides a (web-based) GUI for its workflows. However, all data to be processed by Galaxy must either be on the server itself or uploaded to a location that is reachable by the server. Galaxy can access cloud storages, but classified data may not be uploaded to such storages as pointed out in the introduction. Additionally, Galaxy requires UNIX knowledge to be installed and does not provide a binary for installation. Galaxy is not cross-platform compatible (Microsoft Windows is supported through WSL but still requires UNIX knowledge). Galaxy users provide Docker containers for Galaxy, where a local storage can be mounted.

Another framework providing similar options is Yabi (Hunter et al., 2012). Yabi is only distributed using a Docker container. 


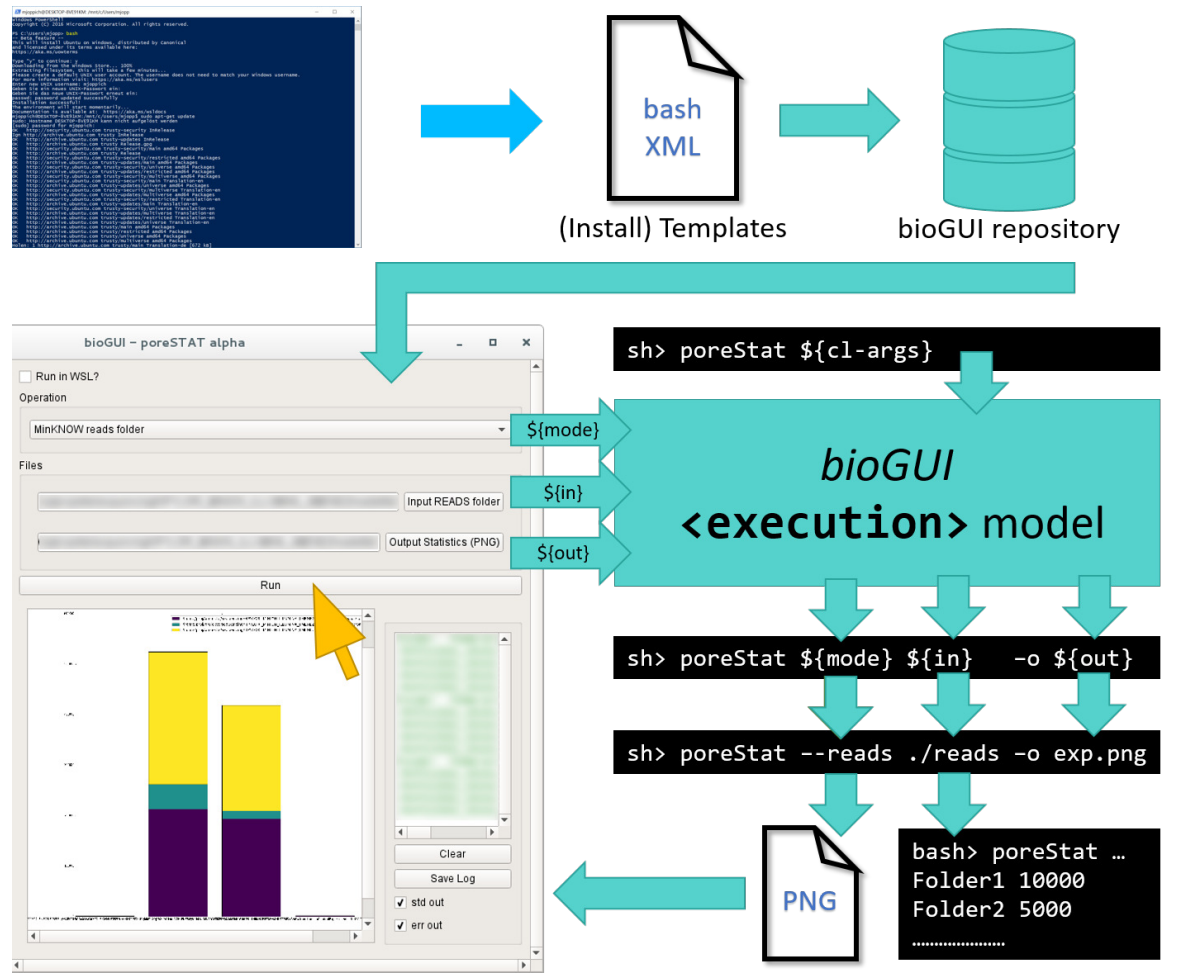

Figure 1. Only little human interaction is needed to run a CL application from a bioGUI template. An (install) template has to be submitted to the bioGUI repository by a developer (blue). The bioGUI application (cyan) allows users (yellow) to download templates or install modules and install and use bioinformatics applications. After the user selected/set the input for the (bioinformatics) application using the GUI, the command-line arguments to run it are constructed from this input. The application's output (text or images) can be directly displayed in bioGUI.

Nextflow \& DolphinNext The combination of Nextflow https://www. nextflow. io/ and DolphinNext https://github.com/UMMS-Biocore/dolphinnext is similar to Galaxy or Yabi. While Nextflow is a DSL for describing general workflows (lacking a GUI definition), DolphinNext provides the web-based user interface (UI) which enables a convenient usage of Nextflow workflows. Nextflow requires a POSIX system architecture and may or may not run on Microsoft Windows using Cygwin (Cygwin, 2019). DolphinNext resembles a lot the Galaxy framework, which can make use of CWL workflows, however, focuses on a deployment in a cluster environment. It is unknown whether or not both systems work on WSL.

Common Workflow Language (CWL) The CWL (Amstutz et al., 2016) is a new standard to define workflows and defines a DSL. In this language, inputs, input-types as well as the corresponding parameters are stored. Additionally, inputs can have a help text included.

Using the bio.tools ToolDog software (Hillion et al., 2017), CWL workflows can be generated and exported for many bioinformatics applications. An advantage of using bio.tools is the automatic annotation and description of input and outputs. Unfortunately, for many packages no CWL workflows have been deposited.

SEED 2 \& BTW In contrast to the previously mentioned tools, SEED 2 (Větrovský et al., 2018) and BTW (Morais et al., 2018) do not focus on running complex workflows in a cluster environment. Instead, these focus on specific tasks which can be run on regular laptop computers. SEED 2 focuses on amplicon high-throughput sequencing data analyses. On the other hand BTW follows the same concept, but focuses on the analysis of marker gene data and does not provide a GUI for this task. SEED 2 provides a GUI to perform the relevant analyses fast and conveniently, while BTW focuses on the usability of UNIX CL tools on Windows. 
RGG \& Autolt RGG was developed as a general GUI framework for R applications (Visne et al., 2009). It uses XML files to specify the input fields for the graphical representation. When the user has set all options, the GUI is translated into an R script for execution. The execution output can also be retrieved from the RGGRunner application. The RGG software is limited to R scripts, but still the authors expressed their hope that providing GUIs for analytical pipelines could "help to bridge the gap between the $\mathrm{R}$ developer community and GUI-dependent users" (Visne et al., 2009).

In contrast to RGG, AutoIt (AutoIt, 2018) is a general automation framework which, similar to bioGUI, allows the definition of a GUI as well as a task that is executed according to this input. In contrast to AutoIt, bioGUI is cross-platform compatible, supports WSL and provides install modules for bioinformatics applications.

Comparison to bioGUI bioGUI is not a classical workflow system like Galaxy, CWL or DolphinNext with Nextflow. bioGUI is not meant to run many tasks nor to run in a cluster environment. Moreover, bioGUI does not share the philosophy of having a (compute) cluster setup to run analyses in a repeated fashion. bioGUI is meant to enable the user to perform bioinformatics analysis at their work place.With bioGUI we aim to provide low effort usage of bioinformatics applications, without the need to setup a complicated environment. Finally this allows to easily compare different methods on collected experimental data.

bioGUI finds its niche as a generalisation of the concepts introduced by Větrovský et al. (2018) and Morais et al. (2018). SEED 2 provides a GUI such that a broad public has access to sophisticated and well-known bioinformatics CL applications in the context of amplicon analysis. Similar concepts, yet differently implemented, are provided by RNA CoMPASS (Xu et al., 2014) for pathogen-host transcriptome analysis or PipeCraft (Anslan et al., 2017). Here custom (web-)UIs let the user interact with their specialised pipelines. RGG (Visne et al., 2009) offers a general GUI framework for R applications only. bioGUI offers a similar framework, which is applicable to any (Unix) application. In both, RGG and bioGUI, users/developers specify the visual elements in a XML file. This XML file is then interpreted and translated into a GUI within an application (RGGRunner or bioGUI, respectively) which also shows the output of the script.

The bioGUI framework extends the concepts presented by RGG and SEED 2, for instance, to general applications, and improves accessibility to these applications by providing install modules.

\section{Use-case study}

One of the main goals we had in mind when developing bioGUI is to create a powerful framework, which is easy-to-use for scientists/users and which does not create significant overhead for the application developer. In order to study this, we introduce two classes of possible users: The first class represents a general user of the software who generally prefers a GUI for performing a research task, e.g. data analysis after sequencing. The second class describes a software developer releasing an application of a new algorithm to solve the alignment of sequencing reads. This class thus depicts a typical developer.

From the two use-cases (see also Appendix A.1) we identify several requirements/goals for bioGUI:

1. installing new programs must be simple and should not require system administrators

2. creating a GUI for a program must not take a lot of time

3. templates must bring a basic GUI to run the programs, output must not be interpreted

4. templates must be saveable for later re-use and reference, and also searchable

5. the system must be lightweight (runtime overhead, disk-space) to even run on laptops

6. installing a program may require additional (protected) external files

Finally, we developed a paper mockup with which we went through the anticipated workflow of the user. We identified several input components and features the bioGUI program has to include (Appendix Figure A1). 


\section{bioGUI approach}

"The accessibility of bioinformatics applications is crucial and a challenge of contemporary biology" (Morais et al., 2018). Particularly the usage of CLIs poses a problem. Since most bioinformatics applications require the execution of commands on the CL for installation (such as for compilation, adding dependencies to the path variable, etc.), we estimate that also the installation poses a problem.

During the use-case study, and interviews with wet-lab scientists without a computational background (Emslander, 2019; Jimenez, 2019), we found two main problems with bioinformatics applications for scientists which we want to address with bioGUI: first the installation of potentially useful applications and second its usage. Both problems have in common, that they are expected to be performed on the CL. A GUI for achieving the respective tasks in bioinformatics (and beyond) is missing.

Especially the first task, installing bioinformatics applications on a user's machine, poses a few problems. Most bioinformatics applications are written for a UNIX operating system, like Linux or Mac OS, while in general Microsoft Windows is the dominant operating system. In order to overcome this problem, bioGUI makes use of WSL on Windows. Even if the user's OS is already Unix-like, using the CL to install software might be strugglesome. Thus, in order to support all users, bioGUI uses a cross-platform approach. bioGUI is developed in C++ using the Qt framework.

The general workflow for any program using bioGUI is shown in Figure 1. Given a CL application, the software developer (blue) writes the specific template in a XML-based DSL and can then make this template available, e.g. in the bioGUI repository (cyan). Such templates can be automatically retrieved by bioGUI. Upon selection of a template by the user, bioGUI displays the input mask as defined in the template. When the user (yellow) has filled in all parameters, the parameters are collected by bioGUI and assembled into CL arguments which are used to execute the original CL-only application. Upon completion, simple results (like text-output or images) can be shown in bioGUI directly, or an external application is opened.

\section{Install Modules}

Install modules are designed to install applications such that bioGUI can access them. Essentially, install modules are bash scripts which allow an automatic installation of applications into a predefined location. For this purpose, install modules receive several arguments from bioGUI when launched, e.g. where to install the application to, the sudo password to fetch packages via a system's package manager (e.g. aptitude, conda, ...), whether the application should be made available to the user via the system's PATH variable, etc .Install modules download and install applications and make them available to the user and bioGUI. However, some applications cannot be simply downloaded, but are distributed by installers. For this purpose, the install module template can be extended by further input fields. These must be specified by bioGUI elements and their values are added to the end of the CL arguments of the install module. An install module can then execute the referenced installer.

Finally, an install module should contain the specification of its bioGUI template and could hard-code the path to the installed application. Other constant values, which can already be derived during the installation (e.g. absolute paths to dependencies), could also be defined in the template during this stage.

\section{bioGUI Templates}

bioGUI templates are the actual end-user-interface to programs. A bioGUI template defines the look and functions of the UI. Thus it can define how the CL-application is called (with corresponding parameters).

Each bioGUI template consists of two parts (Appendix Figure A2). The first part (<window $>$ model) defines the visual appearance of the GUI. The second part (<execution> model) defines the processing logic of the template. Input values from the GUI components are collected and assembled (e.g. pre-/postprocessing steps) to call command-line applications. As part of this assembly, input values from the GUI may be transformed using (multiple) predefined nodes. Concatenations are possible using the $\langle$ add $>$ node, and constant values can be inserted using the <const $>$ node. System environment properties, such as the operating system, the computer's IP address or specific directories can be collected using the <env $>$ node. If the regular nodes are not sufficient, e.g. because more complex string manipulations should be made (see use-case study), script nodes may also accept functions written in LUA (Lua, 2019) or JavaScript (JavaScript, 2019).

In general, the execution part infers a network with inputs (e.g. GUI elements, other nodes within the execution part) and actions (if, add, ...). For example, the execution network for an application with many sub-commands is exemplarily shown in Appendix Figure A3. 
The time to template varies with the application as well as the number of options to be included. A simple template, like the one for MS-EmpiRe (Ammar et al., 2019), can be created within 10 minutes. More comprehensive templates, like the one for HISAT2, usually take about 30 minutes. Time can be saved if only the most important command line options are shown in the GUI. This can be achieved by adding an "optional parameters" input field, where users can insert CL arguments themselves. This is, for instance, shown in the wtdbg2 (Ruan and Li, 2019) and spades (Bankevich et al., 2012) templates. Adding the install part to a template usually can be done within 15-30 minutes, depending on how detailed the build process is documented. The creation of an install module thus takes approximately one hour.

\section{bioGUI Integration with CWL and argparse}

CWL (Amstutz et al., 2016) only describes the CL workflow and neither provides a GUI nor means to install the desired tool. Due to this more general specification, CWL fits most problems, but specific annotations of inputs, explanations or the embedding of images is not supported in CWL.

While developers can always create templates manually, bioGUI supports developers by offering a template generator from CWL templates or python3 argparse CL parsers. Since there are already many CWL templates available for bioinformatics CL applications, CWL files can be used as a base to automatically generate bioGUI templates from. Using the bioGUI template generator for argparse, it is also possible to automatically generate templates from CWL files (making use of the cwl2argparse program provided by CWL). Our generator takes as input the argparse parser or CWL file and creates input elements for all elements. In case the type of an input is unclear or not supported, the generator falls back to a regular text-input element.

\section{RESULTS}

\section{bioGUI Templates}

Currently more than 25 (install) modules exist for bioGUI. These represent basically three groups of bioinformatics tasks: next-generation sequencing (NGS) data analysis and transcriptomics, long read sequencing analysis and assembly as well as more general sequence analysis. In general these install modules will install the respective application on the local machine. The Circlator (Hunt et al., 2015) template allows to pull and use the corresponding Docker image. The available tools, as well as their respective categorization, are listed in Table 1.

\section{Benchmarking bioGUl templates}

Our benchmark comprises of four tasks. The first task is to assemble a bacterial genome from Oxford Nanopore long reads, for which the Minimap2 (Li, 2018)/miniasm (Li, 2016)/Racon (Vaser et al., 2017) pipeline (available as install module from bioGUI) is used. The second task is the quantification of reads from a yeast mRNA sequencing project using Oxford Nanpore Reads and Illumina Reads (EMBL ENA studies PRJNA398797(MinION) and SAMN00849440(Illumina) ). The quantification is performed using featureCounts from the subread package (Liao et al., 2014). The third task uses these results to compute differential gene expression. Differential gene expression analysis is performed using MS-EmpiRe in R (install module available, Ammar et al. (2019)). Finally the fourth task uses RNAhybrid to predict miRNA binding sites (1978 murine miRNAs) in 170 sequences of each 200nt.

The results are shown in Table 2. The given runtimes are wall clock times. The peak RAM consumption has been sampled from the process viewer on the given operating systems (Task Manager on Windows, top on Linux and Mac OS).

\section{DISCUSSION}

bioGUI is a framework for easy GUI-based usage of CL applications in the life sciences. Using bioGUI, high-quality CL applications can be made accessible to as many researchers as possible. This is achieved by lowering the hurdles to overcome for using bioinformatics applications, particularly on Windows.

\section{Use-case analysis}

Our use-case analysis (Appendix Section A.1) has revealed several requirements for bioGUI (see Methods Section) to enable the user to perform the sequencing analysis and to allow the developer a fast template creation (Figure 2). 


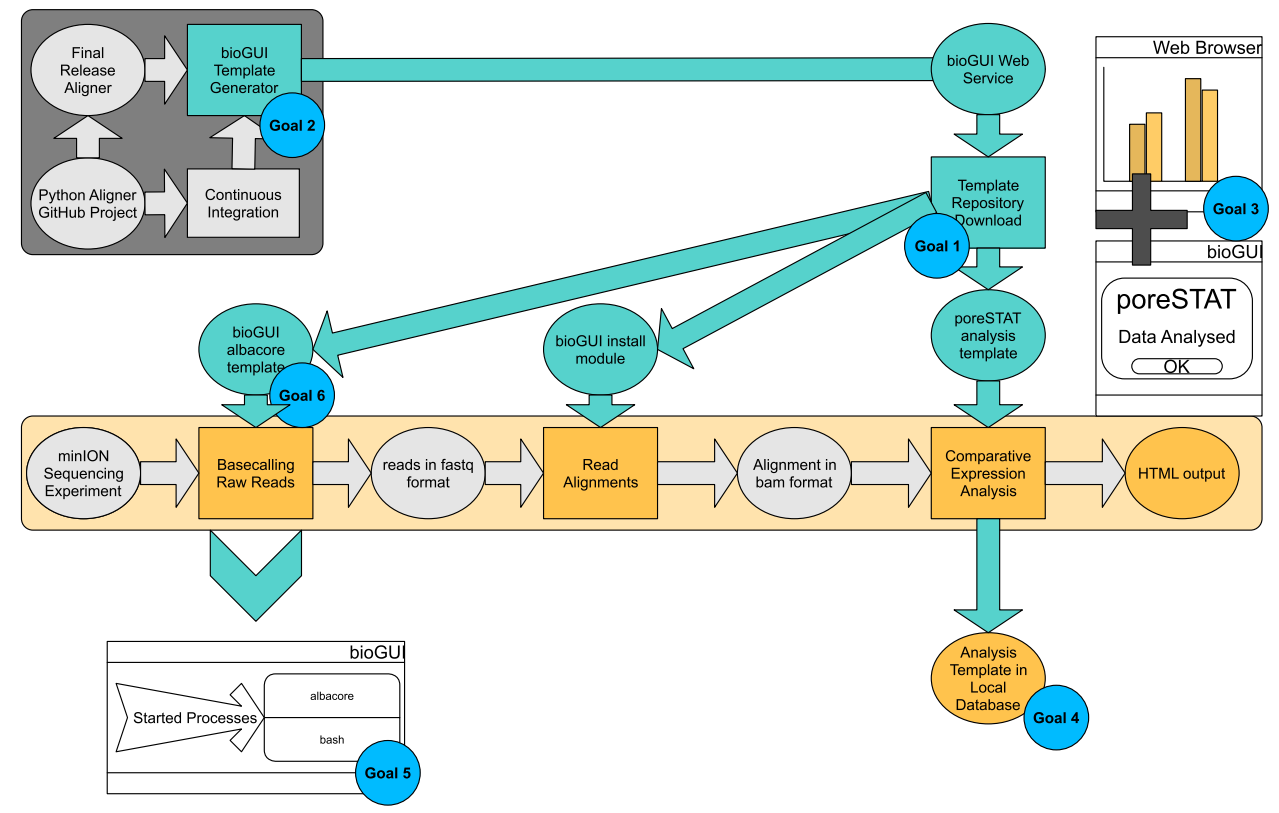

Figure 2. bioGUI use-case study, from a developer's and user's perspective, performed on an exemplary RNAseq analysis workflow. The dark-gray underlayed tasks represent the developer's tasks, and the bright-yellow part represents the analysis pipeline the user wants to execute. Tasks requiring user-action are shown as rectangles and intermediate results are shown in ellipses. Cyan ellipses denote solutions/results (e.g. template repository) offered by bioGUI. bioGUI starts sub-processes for each task, such that the overhead for any started processes is as small as possible. Upon finishing a task or pipeline, bioGUI can display a notification and open generated output. 
Table 1. List of available templates and install modules (starting with Install) for bioGUI.

\begin{tabular}{|c|c|c|c|}
\hline Module Name & Task & $\begin{array}{l}\text { Install M } \\
\text { WSL \& } \\
\text { Ubuntu }\end{array}$ & Mac OS \\
\hline First Time Mac OS Setup & Initialisation & - & $\checkmark$ \\
\hline First Time Ubuntu/WSL/apt-get Setup & Initialisation & $\checkmark$ & - \\
\hline Install Ballgown v1.0.1 (Pertea et al., 2016) & NGS transcriptomics & $\checkmark$ & \\
\hline Install Bowtie1 (Langmead et al., 2009) & NGS & $\checkmark$ & \\
\hline Install Bowtie2 v2.2.9 (Langmead and Salzberg, 2012) & NGS & $\checkmark$ & $\checkmark$ \\
\hline Install bwa v0.7.17 (Li and Durbin, 2009)) & NGS & $\checkmark$ & $\checkmark$ \\
\hline Install canu (github, Koren et al. (2017) & Assembly & $\checkmark$ & \\
\hline Install featureCounts (Liao et al., 2014) & NGS transcriptomics & $\checkmark$ & $\checkmark$ \\
\hline Install glimmer302b (Delcher et al., 2007) & Genome Annotation & $\checkmark$ & \\
\hline Install graphmap (Sović et al., 2016) & Long Read Sequencing & $\checkmark$ & $\checkmark$ \\
\hline Install albacore (pip wheel, ONT) & Long Read Sequencing & $\checkmark$ & \\
\hline Install guppy (linux tar.gz, ONT) & Long Read Sequencing & $\checkmark$ & \\
\hline Install hisat2 (Kim et al., 2019) & NGS transcriptomics & $\checkmark$ & $\checkmark$ \\
\hline Install hmmer-3.1b2 (Wheeler and Eddy, 2013) & Sequence Analysis & $\checkmark$ & \\
\hline Install jellyfish-2.2.6 (Marçais and Kingsford, 2011) & NGS & $\checkmark$ & \\
\hline Install minimap $2 /$ miniasm/racon (github) & Assembly (long-read) & $\checkmark$ & $\checkmark$ \\
\hline Install MS-EmpiRe (Ammar et al., 2019) & NGS transcriptomics & $\checkmark$ & $\checkmark$ \\
\hline Install PureSeqTM Wang et al. (2019) & Sequence Analysis & $\checkmark$ & \\
\hline Install rMATS-3.2.5 (Shen et al., 2014) & NGS transcriptomics & $\checkmark$ & \\
\hline Install rnahybrid (Rehmsmeier et al., 2004) & Sequence Analysis & $\checkmark$ & $\checkmark$ \\
\hline Install RSEM v1.3.0 (Li and Dewey, 2014) & NGS transcriptomics & $\checkmark$ & \\
\hline Install samtools-1.3.1 (Li et al., 2009) & NGS & $\checkmark$ & $\checkmark$ \\
\hline Install SPAdes v3.13.0 (Bankevich et al., 2012)) & Assembly (hybrid) & $\checkmark$ & $\checkmark$ \\
\hline Install StringTie v1.3.0 (Pertea et al., 2016) & NGS transcriptomics & $\checkmark$ & \\
\hline Install Top Monitor (ssh example) & Technical Demo & $\checkmark$ & $\checkmark$ \\
\hline Install Trimmomatic v0.36 (Bolger et al., 2014) & NGS & $\checkmark$ & \\
\hline Install wtdbg2 (Ruan and Li, 2019) & Assembly (long-read) & $\checkmark$ & $\mathrm{x}$ \\
\hline Template Circlator (Hunt et al., 2015) & Assembly & $\checkmark$ & $\checkmark$ \\
\hline
\end{tabular}

Tools marked with $\checkmark$ provide an install module for the operating system of the respective column.

An easy installation (goal 1) is given through the availability of install modules, which can be downloaded from the bioGUI repository and started via a GUI. These also allow additional inputs (e.g. python wheels for albacore, goal 6).

The install modules combine the installation of an application and the creation of the actual GUI template. If the developers employ automatic testing of their software (e.g. build checks with Travis (https://travis-ci.org/)), the install part resembles the Travis container setup (goal 2): dependencies and the application itself are installed into an operating system. Even if not, most bioinformaticians extensively use Ubuntu and/or bash-scripts. Thus writing a script to install dependencies is not a significantly hard workload. We have reached a seamless and time-efficient creation of templates using an XML-based domain-specific language. XML is particularly helpful as it allows to specify hierarchies and attributes to objects. Using our template generator for CWL and python3-argparse, bioGUI templates can be created even faster (goal 2). The templates are highly flexible in the creation of CL parameters, also due to providing script nodes. By providing install modules and templates, high-quality open-source bioinformatics applications become more accessible to the community.

The bioGUI application is cross-platform compatible and only requires a few MB of disk space (goal 5). bioGUI implements several possibilities to execute applications (see Appendix Figure A4). In general, the only runtime overhead involved is the creation of a bash-process which starts the actual program with the assembled CL arguments (goal 5). bioGUI, being a local stand-alone application, has the possibility 
Table 2. Benchmarking results for the 4 selected tasks (see Benchmarking bioGUI templates within the Results section) on the described hardware (see Appendix Table A1). All runs are started via bioGUI.

\begin{tabular}{lllllllll}
\hline \multirow{2}{*}{ Task } & \multicolumn{2}{l}{ Linux Server } & \multicolumn{2}{l}{ Lenovo T470 } & \multicolumn{2}{l}{ Surface Book } & \multicolumn{2}{l}{ MacBook Air } \\
& Time & Peak RAM & Time & Peak RAM & Time & Peak RAM & Time & Peak RAM \\
\hline Assembly & $10: 12 \mathrm{~min}$ & $6.8 \mathrm{~GB}$ & $23: 00 \mathrm{~min}$ & $6.5 \mathrm{~GB}$ & $30: 00 \mathrm{~min}$ & $6.5 \mathrm{~GB}$ & $44: 30 \mathrm{~min}$ & $6.5 \mathrm{~GB}$ \\
\hline featureCounts (MinION) & $00: 38 \mathrm{~min}$ & $20 \mathrm{MB}$ & $00: 54 \mathrm{~min}$ & $18 \mathrm{MB}$ & $01: 12 \mathrm{~min}$ & $18 \mathrm{MB}$ & $01: 30 \mathrm{~min}$ & $18 \mathrm{MB}$ \\
featureCounts (Illumina) & $01: 13 \mathrm{~min}$ & $28 \mathrm{MB}$ & $01: 41 \mathrm{~min}$ & $25 \mathrm{MB}$ & $02: 02 \mathrm{~min}$ & $25 \mathrm{MB}$ & $02: 30 \mathrm{~min}$ & $25 \mathrm{MB}$ \\
\hline DE quantification (MinION) & $00: 19 \mathrm{~min}$ & $0.7 \mathrm{~GB}$ & $00: 25 \mathrm{~min}$ & $0.6 \mathrm{~GB}$ & $00: 28 \mathrm{~min}$ & $0.6 \mathrm{~GB}$ & $00: 42 \mathrm{~min}$ & $0.6 \mathrm{~GB}$ \\
DE quantification (Illumina) & $00: 14 \mathrm{mins}$ & $0.5 \mathrm{~GB}$ & $00: 19 \mathrm{~min}$ & $0.4 \mathrm{~GB}$ & $00: 20 \mathrm{~min}$ & $0.4 \mathrm{~GB}$ & $00: 31 \mathrm{~min}$ & $0.4 \mathrm{~GB}$ \\
\hline RNAhybrid & $07: 35 \mathrm{~min}$ & $19 \mathrm{MB}$ & $23: 00 \mathrm{~min}$ & $18 \mathrm{MB}$ & $13: 00 \mathrm{~min}$ & $18 \mathrm{MB}$ & $16: 55 \mathrm{~min}$ & $18 \mathrm{MB}$ \\
\hline
\end{tabular}

to target both, locally installed and web-based applications, reachable within a controlled environment and with large data. In addition, bioGUI also supports the use of Docker containers, for cases where all other options fail.

The UI can be made easily understandable (goal 3). Using text-labels, the user can get help on inputs (if specified by the developer), links can be used to provide further information and most importantly tool-tips could also hint the user to which information is needed at a certain step.

Finally, filled out templates can be saved via the Save Template button in bioGUI, and all available templates can be filtered (goal 4). This enables to keep track of performed analyses, and makes results more reproducible, because parameters are saved. Having the possibility to save templates also allows to easily repeat analysis with the same parameters. Additionally, using the bioGUI repository, templates can easily be shared among users, making it easier to standardize runs among different users or even institutes.

An anonymous survey (with 10 participants) about common problems in using bioinformatics software has been conducted among colleagues $(n=4)$ and under-graduate students or collaborators $(n=6$, short: collaborators). The results are available in the Appendix Section A.8.

We asked "What were the most struggle-some tasks in accessing and using the software?", referring to recently used bioinformatics software by the participants. 8 of the 10 participants answered that finding parameters or using the software has been strugglesome. This shows that the selected goals for bioGUI address actual problems faced by both experts and regular users. We further asked the participants to install and use graphmap (Sović et al., 2016), which has been selected because it is reasonably easy to install and use. First the participants were asked to install the tool using the CLI as well as via bioGUI. For this task, all instructions have been provided. The question "Has the installation process been easy?" ( $0=\mathrm{No}, 5=$ Yes) has been answered with an average score of 4.4 for the CLI and 4.8 for bioGUI. Then the participants were asked to align the given reads against a given reference genome - this time without giving the instructions. Again we asked "Has it been easy to align the reads?" ( $0=\mathrm{No}, 5=\mathrm{Yes})$. Here the CLI scored a 3.5 on average and bioGUI a 4.9. (Appendix Figure A6). This coincidences with the answer to our question "Overall: Which interface was easier to use in your opinion?" (0=CLI, $5=$ GUI). Here the average score has been 4 . Bioinformaticians and collaborators answered differently: the average bioinformatician has been undecided on which interface has been easier to use (average 3), but non-bioinformaticians preferred the GUI over the CLI (average 4.5).

The survey indicates that there are problems with bioinformatics software regarding installation and usage of CLI tools. These problems can be reduced by providing a GUI for these programs. The more experienced a user is on the CL, the less impact a GUI has. But particularly for non-experts on the CL, a GUI makes it easier to use a program.

\section{bioGUI repository}

We provide a repository of preconfigured templates on our website (Appendix Figure A5), where authors and users can search for and browse existing templates, or submit new ones. bioGUI can access uploaded templates and save them directly for use. Specifically for WSL and Ubuntu users install modules are provided, which take care for dependency resolution and install applications (locally) into the user's home. This currently works in any environment using the aptitude (apt) package manager, but users can submit templates which also support other environments, since install modules are versatile bash scripts. On Mac OS, some install modules support Homebrew for template installation. Install modules download and 
potentially severely alter a system (especially if the sudo password is supplied). Thus, submitted install modules are manually curated and are only accessible when no security threat has been identified.

The major goal of bioGUI is to enable any scientist to use bioinformatics applications. While we extend the repository on a regular basis depending on our own use, users can also request new templates for applications relevant to them.

\section{Availability \& Extensibility}

bioGUI is open-source software and users or institutions can either use our global bioGUI repository or deploy a custom repository, e.g. one which is only reachable within an institution.

bioGUI is available on GitHub https://github.com/mjoppich/bioGUI. Both source code as well as pre-built binary distributions (for Microsoft Windows, Linux and Mac OS) are available. While bioGUI will run on any Linux distribution, install modules currently use mainly aptitude as package manager (e.g. Ubuntu, debian-based distributions). If used on Windows, the same applies for the used WSL-application (Ubuntu 18.04 is recommended). bioGUI has been tested on Microsoft Windows 10, build 17763. On Mac OS bioGUI uses Homebrew (https://brew.sh/) to install dependencies. Homebrew does not support a silent, non-interactive installation: the user has to install Homebrew before running the First time setup for Mac OS install module which will install the most common dependencies.

While a number of use cases and corresponding components are already included in bioGUI, we encourage users to contribute on GitHub by either pushing their own extensions, or opening feature requests. Further documentation (installation \& setup guide, how to write templates) is also available via ReadTheDocs (https://biogui.readthedocs.io/en/latest/index.html).

\section{Benchmarking bioGUI templates}

bioGUI starts a sub-process for each program executed. Thus, the only overhead created by bioGUI itself is the one for running the GUI, which creates less than $1 \%$ CPU usage, allocates less than 50MB and only performs IO operations when loading a new template (assessed via Sysinternals Process Explorer (Microsoft Sysinternals, 2019)).

Nonetheless we have been interested in demonstrating that many bioinformatics tasks do not require a dedicated server setup but can be performed on regular laptop computers. We thus benchmark four typical tasks performed using bioGUI.

The selected tasks allow a good overview of different demands: Tasks 1 (assembly) and 3 (differential expression analysis) are CPU-bound tasks, while tasks 2 (feature counting) and 4 (miRNA target prediction) are IO-bound. Particularly task 2 has a high load of read operations, and task 4 has a high demand of write operations. We compare these tasks on a dedicated (Linux) server, one (rather) powerful Lenovo T470p laptop computer, one Surface Book laptop computer (resource-wise a typical laboratory laptop) as well as one MacBook Air. The computer specifications are listed in the Appendix Table A1 and results are shown in Table 2. Even though we have not included the alignment of the Illumina yeast reads in this benchmark, it should be noted that this task also runs well on laptop computers. On the Lenovo laptop the alignment of the SRR453566 sample, consisting of 5,725,730 paired reads, has a peak RAM consumption of 34MB and took 13:50 min, while the Surface Book is even faster at less than $8 \mathrm{~min}$. This presumably can be explained due to different SSD speeds.

The results in Table 2 show that even (computational power-wise) lower-end computers can run bioinformatics tools. More interestingly these results show that the WSL allows the execution of interesting bioinformatic tools. It can be seen that WSL is slow for IO operations, but has a comparable speed for in-memory operations. Particularly tools requiring a lot of IO are considerably faster on the Linux Server (Assembly, RNAhybrid), while the computational expensive tools like MS-EmpiRe (Ammar et al., 2019) and featureCounts (Liao et al., 2014) run within similar times.

\section{CONCLUSION}

The bioGUI framework makes it easy to develop, provide and use GUIs for CL applications. Particularly for non-computer experts, using CLIs is strugglesome. Providing a GUI and/or install modules increases accessibility to high-quality bioinformatics applications for these users. bioGUI creates a cross-platform GUI experience for many open-source bioinformatics applications. In particular, bioGUI enables the deployment of academic bioinformatics applications to Microsoft Windows workstations and laptops, but also to Linux or Mac OS. 
The separation of the GUI components and the program logic allows the creation of templates in two steps. First, the template developer adds input elements to the window and, second, assembles these inputs according to the needs of the application back into CL arguments. This way almost any command-line application can be used with a GUI, enabling many more researchers to use open-source tools. Providing install modules to make UNIX applications available to Microsoft Windows users (via WSL) supports this goal.

bioGUI can not always replace dedicated GUIs. A tailored UI will still be more usable and userfriendly than any generic solution can be. We experienced this in our use-case: certain tasks (e.g. selecting options) require special solutions, let alone from displaying or interpreting the results. However, especially with the install module concept we aim to provide a seamless installation and create the possibility to run CL applications by all scientists. Using the bioGUI framework, simple GUIs can be constructed. But these simple GUIs already help to make bioinformatic tools more accessible by making execution and usage of these tools more comfortable.

Using bioGUI it becomes a simple exercise to use supported CL applications. Currently, there are already install modules and templates for more than 25 applications in our bioGUI repository. bioGUI lowers the burden to use excellent applications, allowing more scientists a better analysis of their data. With bioGUI it must not be first understood how to use and navigate on the CL, instead, the focus is set on the applications, its method and parameters, and finally the data.

\section{ACKNOWLEDGMENTS}

We thank Luisa Jimenez and Gergely Csaba for their valuable input on how important GUIs are and how to improve this software, as well as for reviewing the manuscript. We thank the participants in our survey for their time. We thank the reviewers for their constructive feedback.

\section{REFERENCES}

Afgan, E., Baker, D., van den Beek, M., Blankenberg, D., Bouvier, D., Čech, M., Chilton, J., Clements, D., Coraor, N., Eberhard, C., Grüning, B., Guerler, A., Hillman-Jackson, J., Von Kuster, G., Rasche, E., Soranzo, N., Turaga, N., Taylor, J., Nekrutenko, A., and Goecks, J. (2016). The Galaxy platform for accessible, reproducible and collaborative biomedical analyses: 2016 update. Nucleic acids research, 44(W1):W3-W10,

Albert, I. (2016). The Biostar Handbook. Albert, Istvan.

Ammar, C., Gruber, M., Csaba, G., and Zimmer, R. (2019). MS-EmpiRe utilizes peptide-level noise distributions for ultra sensitive detection of differentially abundant proteins. bioRxiv, page 514000.

Amstutz, P., Andeer, R., Chapman, B., Chilton, J., Crusoe, M. R., Valls Guimer, R., Carrasco Hernandez, G., Ivkovic, S., Kartashov, A., Kern, J., and al., E. (2016). Common Workflow Language, draft 3.

Anslan, S., Bahram, M., Hiiesalu, I., and Tedersoo, L. (2017). PipeCraft: Flexible open-source toolkit for bioinformatics analysis of custom high-throughput amplicon sequencing data. Molecular Ecology Resources, 17(6):e234-e240.

AutoIt (2018). AutoIt Scripting Language.

Bankevich, A., Nurk, S., Antipov, D., Gurevich, A. A., Dvorkin, M., Kulikov, A. S., Lesin, V. M., Nikolenko, S. I., Pham, S., Prjibelski, A. D., Pyshkin, A. V., Sirotkin, A. V., Vyahhi, N., Tesler, G., Alekseyev, M. A., and Pevzner, P. A. (2012). SPAdes: a new genome assembly algorithm and its applications to single-cell sequencing. Journal of computational biology : a journal of computational molecular cell biology, 19(5):455-77.

Bolger, A. M., Lohse, M., and Usadel, B. (2014). Trimmomatic: a flexible trimmer for Illumina sequence data. Bioinformatics, 30(15):2114-2120.

Cygwin (2019). Cygwin. https: / /www . cygwin. com/, Last accessed 2019-09-22.

Delcher, A. L., Bratke, K. A., Powers, E. C., and Salzberg, S. L. (2007). Identifying bacterial genes and endosymbiont DNA with Glimmer. Bioinformatics (Oxford, England), 23(6):673-9.

Emslander, Q. (2019). Personal Communication.

Grabherr, M. G., Haas, B. J., Yassour, M., Levin, J. Z., Thompson, D. A., Amit, I., Adiconis, X., Fan, L., Raychowdhury, R., Zeng, Q., Chen, Z., Mauceli, E., Hacohen, N., Gnirke, A., Rhind, N., di Palma, F., Birren, B. W., Nusbaum, C., Lindblad-Toh, K., Friedman, N., and Regev, A. (2011). 
Trinity: reconstructing a full-length transcriptome without a genome from RNA-Seq data. Nature Biotechnology, 29(7):644-52.

Gymrek, M., McGuire, A. L., Golan, D., Halperin, E., and Erlich, Y. (2013). Identifying personal genomes by surname inference. Science, 339(6117):321-4.

Hillion, K.-H., Kuzmin, I., Khodak, A., Rasche, E., Crusoe, M., Peterson, H., Ison, J., and Ménager, H. (2017). Using bio.tools to generate and annotate workbench tool descriptions. F1000Research, 6:2074.

Hosny, A., Vera-Licona, P., Laubenbacher, R., and Favre, T. (2016). AlgoRun, a Docker-based packaging system for platform-agnostic implemented algorithms. Bioinformatics, 32(15):2396-2398.

Hunt, M., Silva, N. D., Otto, T. D., Parkhill, J., Keane, J. A., and Harris, S. R. (2015). Circlator: Automated circularization of genome assemblies using long sequencing reads. Genome Biology, 16(1):294.

Hunter, A. A., Macgregor, A. B., Szabo, T. O., Wellington, C. A., and Bellgard, M. I. (2012). Yabi: An online research environment for grid, high performance and cloud computing. Source Code for Biology and Medicine, $7: 1$

JavaScript (2019). ECMAScript 2018 Language Specification. https://www. ecma-international .org/ecma-262/9.0/, Last accessed 2019-09-22.

Jimenez, L. (2019). Personal Communication.

Kim, D., Paggi, J. M., Park, C., Bennett, C., and Salzberg, S. L. (2019). Graph-based genome alignment and genotyping with HISAT2 and HISAT-genotype. Nature Biotechnology, 37(8):907-915.

Koren, S., Walenz, B. P., Berlin, K., Miller, J. R., Bergman, N. H., and Phillippy, A. M. (2017). Canu: Scalable and accurate long-read assembly via adaptive $\kappa$-mer weighting and repeat separation. Genome Research, 27(5):722-736.

Langmead, B. and Salzberg, S. L. (2012). Fast gapped-read alignment with Bowtie 2. Nature methods, 9(4):357-9.

Langmead, B., Trapnell, C., Pop, M., and Salzberg, S. L. (2009). Ultrafast and memory-efficient alignment of short DNA sequences to the human genome. Genome Biology, 10(3):R25.

Li, B. and Dewey, C. N. (2014). RSEM: Accurate transcript quantification from RNA-seq data with or without a reference genome. Bioinformatics: The Impact of Accurate Quantification on Proteomic and Genetic Analysis and Research, 12(1):41-74.

Li, H. (2016). Minimap and miniasm: Fast mapping and de novo assembly for noisy long sequences. Bioinformatics, 32(14):2103-2110.

Li, H. (2018). Minimap2: pairwise alignment for nucleotide sequences. Bioinformatics, 34(18):30943100 .

Li, H. and Durbin, R. (2009). Fast and accurate short read alignment with Burrows-Wheeler transform. Bioinformatics, 25(14):1754-1760.

Li, H., Handsaker, B., Wysoker, A., Fennell, T., Ruan, J., Homer, N., Marth, G., Abecasis, G., and Durbin, R. (2009). The Sequence Alignment/Map format and SAMtools. Bioinformatics (Oxford, England), 25(16):2078-9.

Liao, Y., Smyth, G. K., and Shi, W. (2014). FeatureCounts: An efficient general purpose program for assigning sequence reads to genomic features. Bioinformatics, 30(7):923-930.

Lua (2019). The Programming Language Lua. https : / /www . lua . org/home. html, Last accessed 2019-09-22.

Marçais, G. and Kingsford, C. (2011). A fast, lock-free approach for efficient parallel counting of occurrences of k-mers. Bioinformatics (Oxford, England), 27(6):764-70.

Microsoft Sysinternals (2019). Process Explorer v16.30. https://docs.microsoft.com/ en-us/sysinternals/downloads/process-explorer, Last accessed 2019-09-22.

Morais, D. K., Roesch, L. F., Redmile-Gordon, M., Santos, F. G., Baldrian, P., Andreote, F. D., and Pylro, V. S. (2018). BTW-Bioinformatics Through Windows: an easy-to-install package to analyze marker gene data. PeerJ, 6:e5299.

Pavelin, K., Cham, J. A., de Matos, P., Brooksbank, C., Cameron, G., and Steinbeck, C. (2012). Bioinformatics meets user-centred design: a perspective. PLoS Computational Biology, 8(7):e1002554.

Pertea, M., Kim, D., Pertea, G. M., Leek, J. T., and Salzberg, S. L. (2016). Transcript-level expression analysis of RNA-seq experiments with HISAT, StringTie and Ballgown. Nature Protocols, 11(9):165067.

Rehmsmeier, M., Steffen, P., Höchsmann, M., and Giegerich, R. (2004). Fast and effective prediction of microRNA/target duplexes. RNA, 10(10):1507-1517. 
Ruan, J. and Li, H. (2019). Fast and accurate long-read assembly with wtdbg2. bioRxiv, page 530972.

Schadt, E. E. (2012). The changing privacy landscape in the era of big data. Molecular Systems Biology, $8(1): 612$.

Shen, S., Park, J. W., Lu, Z.-X., Lin, L., Henry, M. D., Wu, Y. N., Zhou, Q., and Xing, Y. (2014). rMATS: Robust and flexible detection of differential alternative splicing from replicate RNA-Seq data. Proceedings of the National Academy of Sciences, 111(51):E5593-E5601.

Smith, D. R. (2013). The battle for user-friendly bioinformatics. frontiers in Genetics, 4:187.

Smith, D. R. (2015). Buying in to bioinformatics: an introduction to commercial sequence analysis software. Briefings in Bioinformatics, 16(4):700-9.

Sović, I., Šikić, M., Wilm, A., Fenlon, S. N., Chen, S., and Nagarajan, N. (2016). Fast and sensitive mapping of nanopore sequencing reads with GraphMap. Nature Communications, 7:11307.

Vaser, R., Sović, I., Nagarajan, N., and Šikić, M. (2017). Fast and accurate de novo genome assembly from long uncorrected reads. Genome Research, 27(5):737-746.

Větrovský, T., Baldrian, P., and Morais, D. (2018). SEED 2: A user-friendly platform for amplicon high-throughput sequencing data analyses. In Berger, B., editor, Bioinformatics, volume 34, pages 2292-2294. Narnia.

Visne, I., Dilaveroglu, E., Vierlinger, K., Lauss, M., Yildiz, A., Weinhaeusel, A., Noehammer, C., Leisch, F., and Kriegner, A. (2009). RGG: a general GUI Framework for R scripts. BMC Bioinformatics, 10(1):74.

Wang, Q., Ni, C.-m., Li, Z., Li, X.-f., Han, R.-m., Zhao, F., Xu, J., Gao, X., and Wang, S. (2019). Efficient and accurate prediction of transmembrane topology from amino acid sequence only. bioRxiv, page 627307.

Wheeler, T. J. and Eddy, S. R. (2013). nhmmer: DNA homology search with profile HMMs. Bioinformatics (Oxford, England), 29(19):2487-9.

Xu, G., Strong, M. J., Lacey, M. R., Baribault, C., Flemington, E. K., and Taylor, C. M. (2014). RNA CoMPASS: A dual approach for pathogen and host. PLoS ONE, 9(2):e89445. 


\section{A APPENDIX}

\section{A.1 Use-cases}

\section{Non-computer expert / Luisa}

Many researchers work in small labs without any significant IT support. The computers in their labs mostly run Microsoft Windows and PhD students often have to bring their own devices (because the institute does not provide such working devices). Particularly in the life sciences, users can profit a lot from existing, open-source software. However, installing major bioinformatics applications on such lab computers often poses a problem: administrators (if existent) have little time to deploy new applications, or there is no support in installing new applications at all. If the users are not computer experts, installing and using command line tools may be strugglesome for them (see Appendix A.8 and Morais et al. (2018)). While there are users that can use the CL efficiently, the cited literature and our personal experience shows that there are many users who do not feel comfortable on the CL. This does not mean that they don't want to learn it or are incapable of learning it, but their focus simply does not lie in learning to use the CL. Instead, they want to get the results for their data, fast, reliably and troubleless. One of these users is Luisa.

Oxford Nanopore Sequencing is becoming more and more popular, and even the sequencing hardware can be found in more and more biological laboratories, like in Luisa's. Particularly important for MinIONsequencing is the post-processing of the actual raw read data. While in previous versions, base-calling has been directly performed in the cloud by Oxford Nanopore, this has now been pushed back to the client side. Thus, despite having the sequencing data on her laptop, Luisa must still retrieve the sequences herself, using, for instance, the Albacore basecaller (if they don't want to rely on LiveBasecalling). Unfortunately, like many bioinformatics packages, the basecaller only comes as a python CL program. Additionally, the download is only available as a python-wheel, which means there is no UI-based setup available. Luisa thus needs assistance for the installation of the python-wheel as well as starting the basecalling process. After the reads are basecalled, reads need to be aligned to a reference genome. While there exist reference genomes in correct format on her lab computer (or can easily be downloaded from the web), the CL program to map the reads is available only from GitHub to be installed from source. Luisa has troubles using the CL to clone the repository, compile and use the CL application.

Luisa does not require a custom analysis of her data, but wants to initially screen her data in a simple, basic and robust analysis. She is mostly busy in her lab, hence an analysis has to be prepared fast, and parameters should be stored for later reference. For this a local searchable database of saved templates is needed.

\section{Software developers}

A developer finished his sequence alignment program. The project is already published on GitHub and in a journal, but only few people start using it. From the issues and feature requests on GitHub it can be seen that mainly other bioinformaticians use the program. Thus the developer decides that the program should be accessible to more researchers and looks for ways to make the program useable by everyone. Since the program is written in python, it is cross-platform compatible per-se. However, it is noticed that domain-experts do not install and use the program. Thus the developer must look for an easy way to distribute the application and make it accessible to more researchers. The developer's time is limited, having other projects waiting. There is also little support for developing GUI from colleagues, as they have different views on the extent of autonomy a wet-lab researcher should have regarding sequencing analysis. 


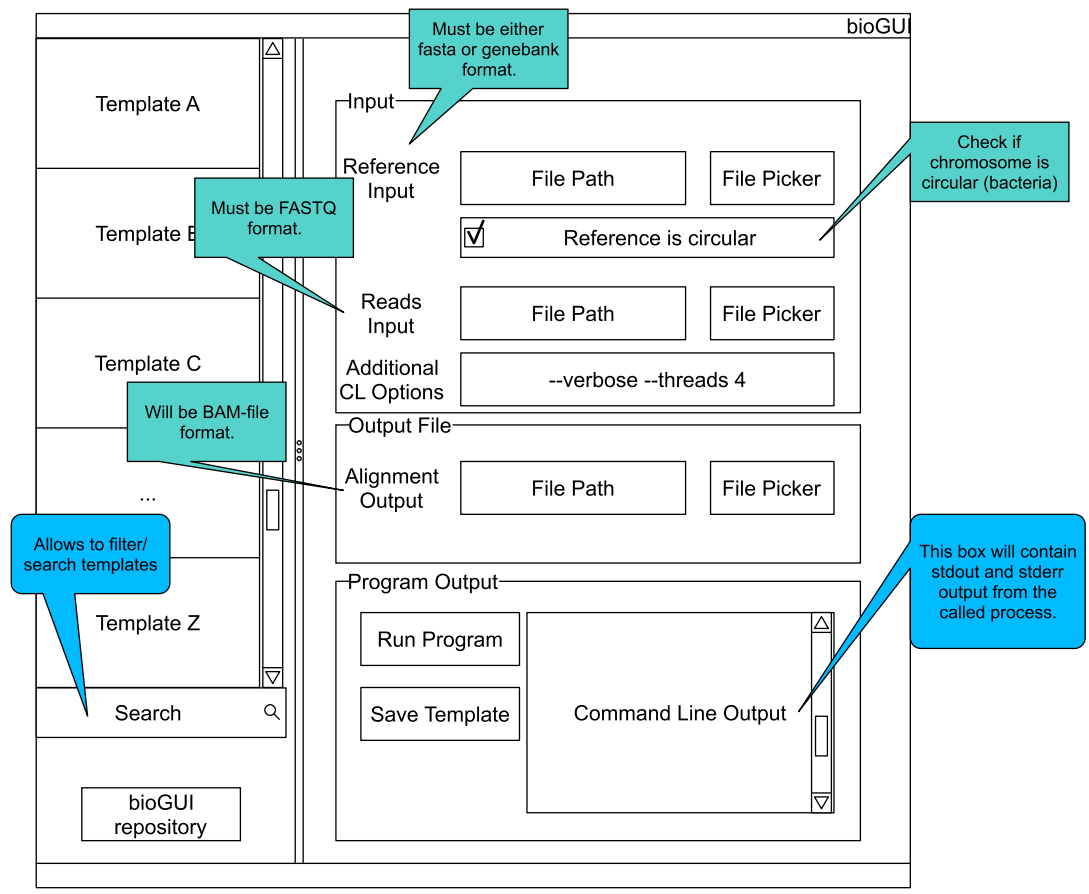

Figure A1. bioGUI mockup showing the elements a template could be made of. The GUI has a searchable list of installed templates as well as a link to our repository of templates. The right side is reserved to the currently displayed GUI template. Here a structured view of the available parameters, as well as hints for filling these, is shown to the user. Finally, the user has the possibility to run the program by clicking a button and to see the program's output.

\section{A.3 Extending templates with script nodes}

Often it is required to perform string-manipulations (e.g. remove file extensions) for CL arguments. For instance, the example below takes as input a HISAT2 index file, and removes the file extension, such that the index will be accepted by HISAT2. For evaluation of this node, the evaluate-function is called with the argv-references as input parameters. The last return-value of the script's call stack is taken as output value of the script-node.

Listing 1. bioGUI script node with LUA function example. Upon evaluating this node, the evaluate function will be called with the arguments listed in the argv attribute of the script node.

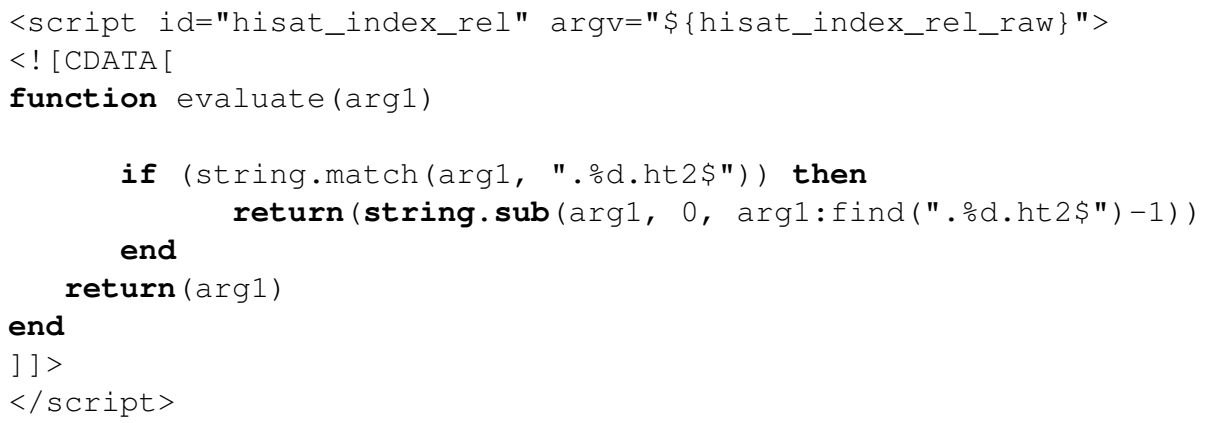




\section{A.4 Evaluating a bioGUI template}

In Appendix Figure A2 the process of assembling a CL call from the shown bioGUI template is explained. First, the creation of the $<$ window $>$ model (dark gray) will be explained, followed by the creation of the CL arguments using the <execution> model (shaded).

The window component consists of four different components, which are grouped in a vertical layout (default for window component). A label describing the input file dialog is placed on the main window, followed by the actual file dialog with ID input. Then a group box with title and a checkable status is created, which contains an output file dialog. Finally, the action button, which starts the CL call assembly and the subprocess of this program, and the text output elements are created.

When the user has entered all desired data, and clicks the action button, the execution phase defined by the execution model will be launched. Therefore the program defined in the execute element is started. For this, the parameters (param) must be assembled. Any text within $\$\{v a r\}$ is interpreted as a reference to a variable var or the value of a GUI element with id var. Thus, the CL is successively assembled. At first the $\$\{$ input $\}$ element is interpreted and retrieves the value from the input file dialog as this element matches the id. Next the $\$\{$ output $\}$ is interpreted. The $\$\{$ output $\}$ refers to an if construct in the execution part, which compares the value of the element with id $o s$ to the string TRUE (which is whether the groupbox is checked). If this value is true, this node evaluates to netcat 192.168.1.100 55025, otherwise to tee -a \{output file path\}. Finally, the program $s h$ is executed with the created CL arguments. For instance, if the group box is checked, the sh ${ }^{-c}$ "cat inputFile | netcat 192.168.1.100 55025" will be executed. A full reference of all input types as well as all execution nodes is available online.

In fact, the evaluation of the execution network resembles the simulation of a petri net (Appendix Figure A3). Each node in the execution network is a place, and its modification/function is the transition, which requires values for all its input places, to generate the output token.

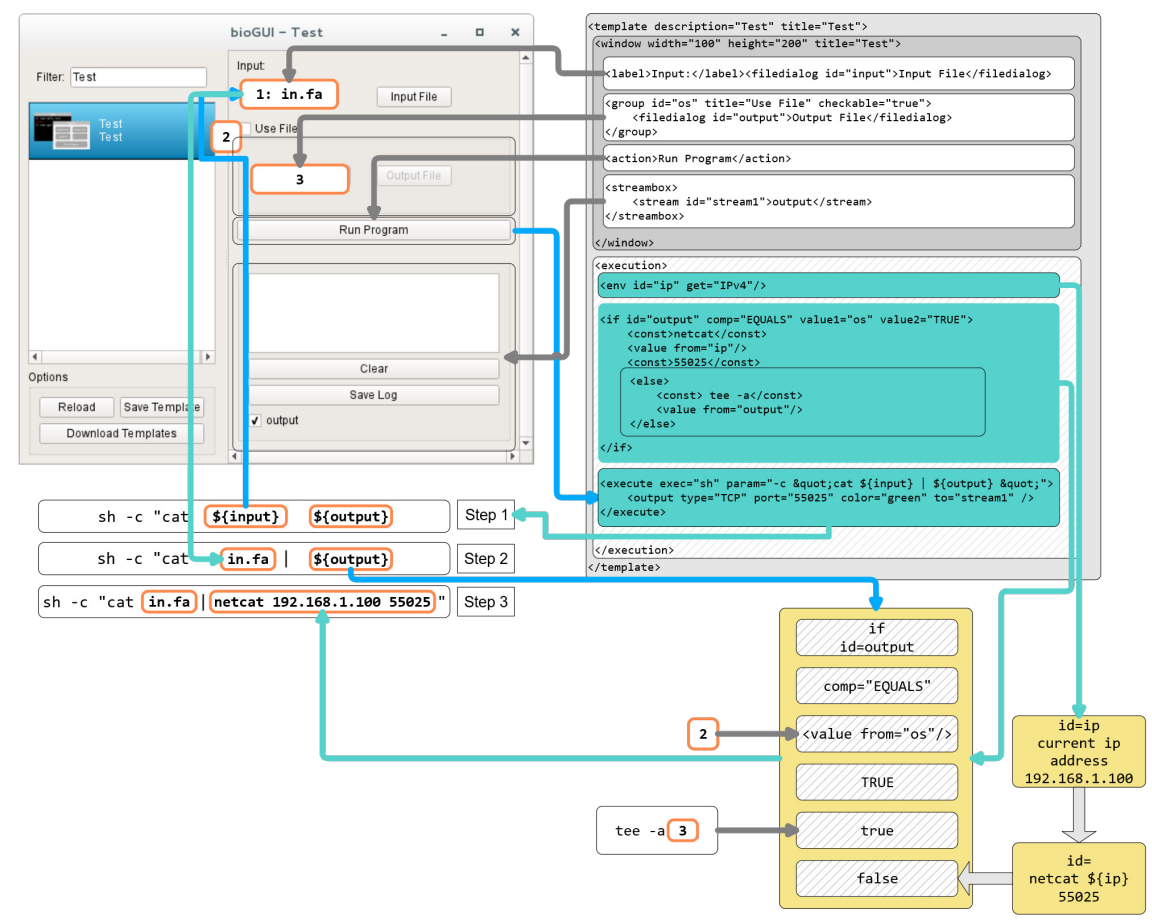

Figure A2. Template construction and evaluation in bioGUI. First, the dark gray window part is evaluated to create the GUI. Once the user clicks the run button, the execution part of the template (shaded) is executed by constructing and starting the assembled system call. This system call is constructed in 3 steps by replacing variables with evaluated terms from the user's input. Blue lines indicate the visual element a returned value (cyan lines) is taken from. Helper/intermediate nodes to be evaluated are shown in light yellow. 
A

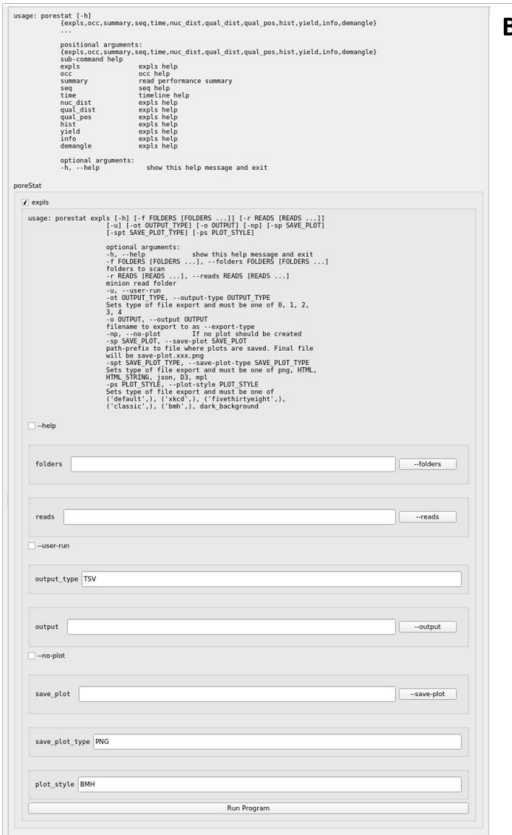

B

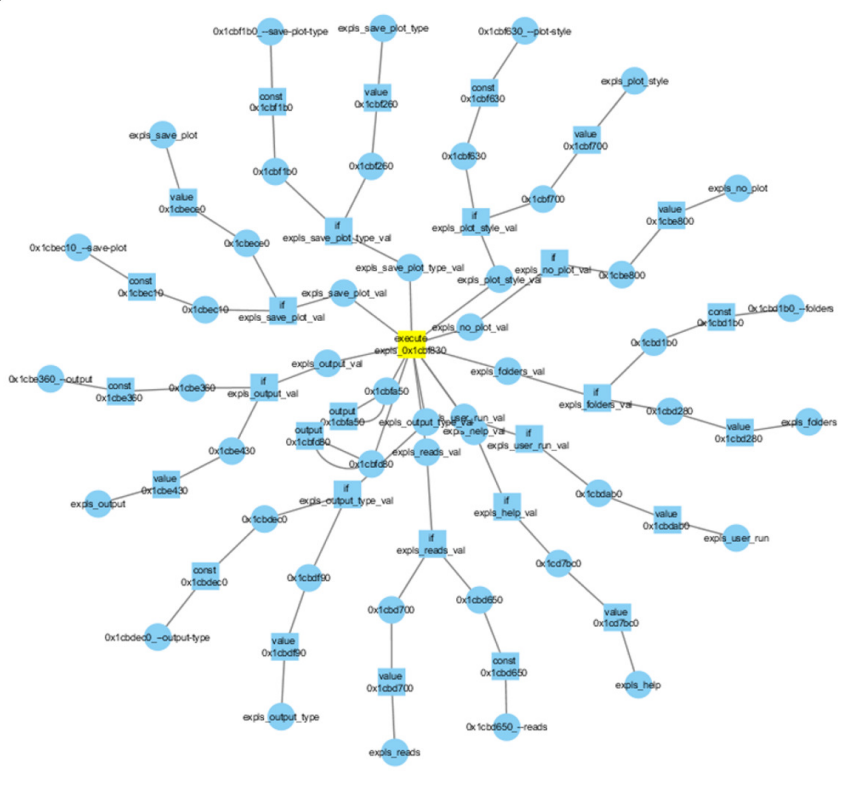

Figure A3. A: An automatically generated bioGUI template from the poreSTAT (Internal tool for minION sequencing analysis) python argument parser. B: The resulting execution network for the bioGUI template shown in (A). The central node represents the fully assembled CL argument (yellow). 


\section{A.5 Running programs via bioGUI}

Program execution via bioGUI can be accomplished via different paths, which are shown in Figure A4. The easiest way is to execute a native program (one that runs natively on the operating system, e.g. Docker). Then all output can be piped to bioGUI to display this to the user. If the host is a Microsoft Windows $10 \mathrm{OS}$, bioGUI can also run Unix programs via WSL. Then the Unix program runs natively in a WSL bash. The resulting output can be transferred to bioGUI via pipes. Of course, for both native and WSL processes, the output can also be transferred via netcat to bioGUI. The transfer of the GUI template within install modules is an example. If a process runs on a remote computer, the output can be transferred to bioGUI also via network, e.g. netcat. Such a process can, for instance, be started by calling $s s h$ from bioGUI with appropriate parameters. Finally bioGUI can also send HTTP POST requests to web-services and accepts an HTTP response as answer. This output can also be displayed by bioGUI.

Since the Docker engine is a local, native process, bioGUI also supports the use of Docker containers. The Circlator template is an example of how this can be implemented.

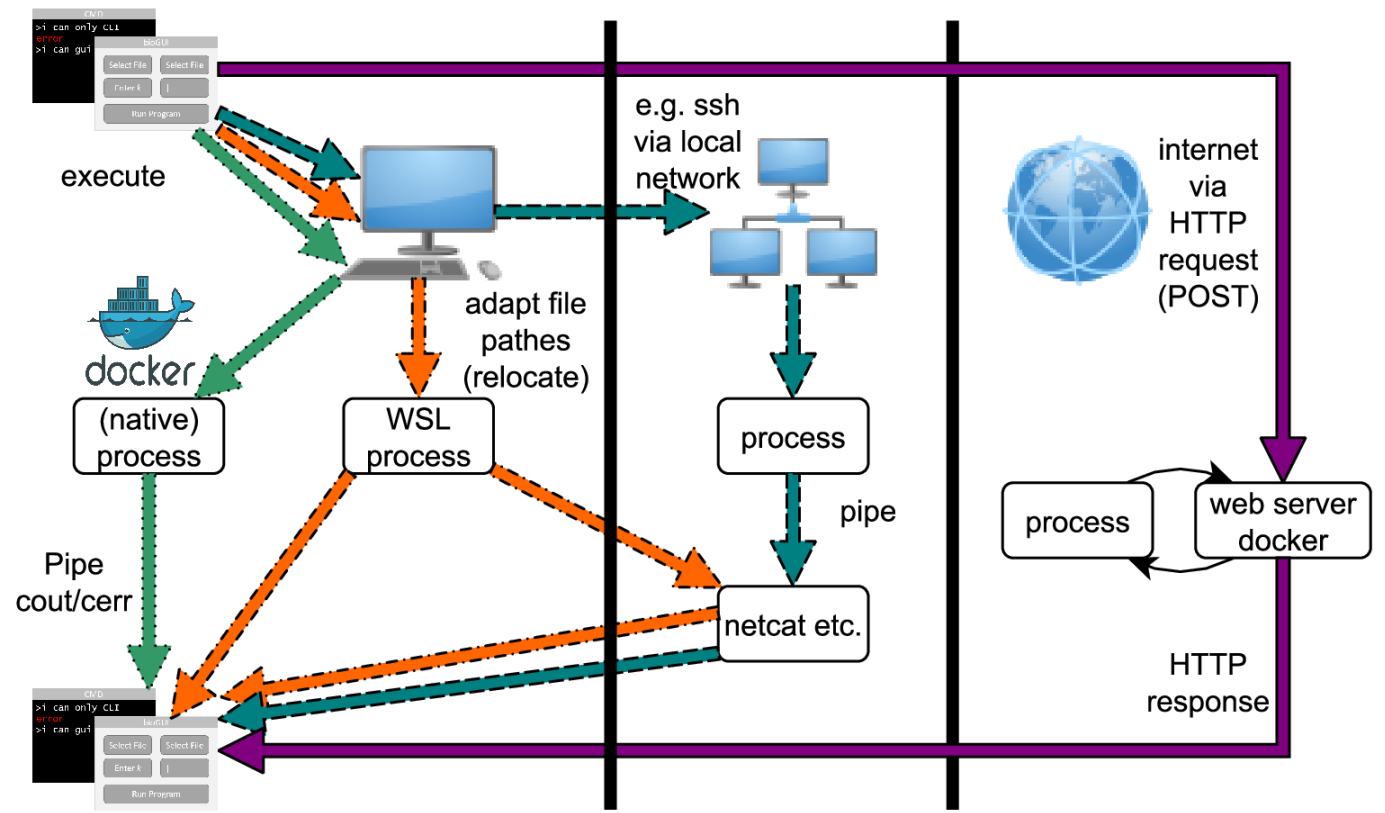

Figure A4. Possibilities for running bioGUI: locally via processes, on a network via ssh or on the web via HTTP request/response. Straight arrow (purple): HTTP execution mode; Dotted arrow (green): Docker execution; Dotdashed arrow(orange): bash/WSL execution; Dashed arrow(cyan): remote/ssh execution 


\section{A.6 Hardware Specification for Benchmarks}

The relevant hardware for benchmarking bioGUI is summarized in Table A1.

Table A1. Hardware used to benchmark bioGUI.

\begin{tabular}{l|l|l|l}
\hline Computer Name & CPU & RAM & Storage \\
\hline Linux Server & $\begin{array}{l}\text { Intel Xeon W-2145 CPU @ 3.70GHz } \\
8 \text { cores (+8 HT cores) }\end{array}$ & $128 \mathrm{~GB}$ & $\begin{array}{l}\text { Samsung SSD 860 } \\
1 \text { TB SSD }\end{array}$ \\
\hline Lenovo Laptop (T470p) & $\begin{array}{l}\text { Intel Core i7-7820HQ @ 2.9GHz } \\
4 \text { cores }+4 \text { HT cores) }\end{array}$ & $32 \mathrm{~GB}$ & $\begin{array}{l}\text { Samsung MZVLB1T0HALR } \\
\text { 1TB SSD }\end{array}$ \\
\hline Microsoft Surface Book & $\begin{array}{l}\text { Intel Core i5-6300U @ 2.4 GHz } \\
2 \text { cores (+2 HT cores) }\end{array}$ & $8 \mathrm{~GB}$ & $\begin{array}{l}\text { Samsung MZFLV128HCGR } \\
128 \mathrm{~GB} \text { SSD }\end{array}$ \\
\hline Apple MacBook Air (mid 2012) & Intel Core i5 @ 1.7GHz & $8 \mathrm{~GB}$ & 128GB SSD \\
\hline
\end{tabular}

614

\section{A.7 Template Access}

$$
\text { A }
$$

bioGUI: Existing Templates

\begin{tabular}{|c|c|c|c|}
\hline Show $10 \cdot$ entries & & & \\
\hline Type & Name & Date & Submitted by \\
\hline Install Template & Jellyish & $2016-07 \cdot 2512: 13.07$ & Markus Joppich \\
\hline Install Template & Glimmer3 & $2016-07.28$ 10:46:22 & Markus Joppich \\
\hline Install Template & hmmer & $2016-07-28$ 10.46:38 & Markus Joppich \\
\hline Template & Trimmomatic & 2016-07-25 12:07:43 & Markus Joppich \\
\hline Template & netcat demo & $2016-07-28$ 10.33.26 & Markus Joppich \\
\hline Template & BLASTN & $2016-07-28$ 10:33:52 & Markus Joppich \\
\hline Template & Bowtie (AlgoRun) & $2016-07-28$ 10:34:37 & Markus Joppich \\
\hline Template & Bowtie (AlgoRun modified) & $2016-07-28$ 10:34:54 & Markus Joppich \\
\hline Template & Install Programs & 2016-07-28 10:37.00 & Markus Joppich \\
\hline
\end{tabular}

в

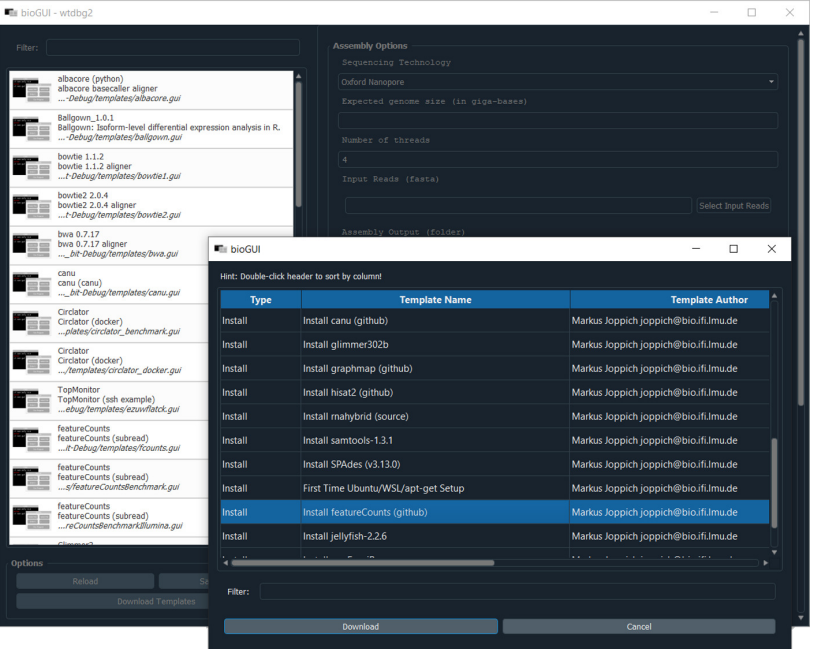

Figure A5. A: On our website a list of already existing templates can be browsed. Besides the description and author, also the type (install module or template) is shown. B: All uploaded templates can be downloaded directly from within bioGUI. bioGUI allows to search in/filter all available install modules and templates. 


\section{A.8 User Survey}

A user survey among 10 participants (4 bioinformaticians, 6 collaborators (consisting of 2 under-graduate bioinformatics students and 4 external collaborators)) has been performed. The derived results are shown in Table A2 and the raw data are shown in Table A3.

Table A2. Derived user survey results from the given answers (Table A3).

\begin{tabular}{l|l|l|l|l|l} 
& $\mathrm{n}$ & median & mean & pvalue & variance \\
\hline Better Interface Bio & 3 & 3 & 3 & & 4 \\
Better Interface Collab & 6 & 4.5 & 4.5 & & 0.3 \\
Better Interface All & 9 & 4 & 4.00 & & 1.75 \\
& & & & & \\
Easy to align CLI & 10 & 3.5 & 3.50 & & 1.833 \\
Easy to align bioGUI & 10 & 5 & 4.90 & 0.0098 & 0.1 \\
Easy to install CLI & 10 & 5 & 4.40 & & 0.711 \\
Easy to install bioGUI & 10 & 5 & 4.80 & 0.2023 & 0.178
\end{tabular}

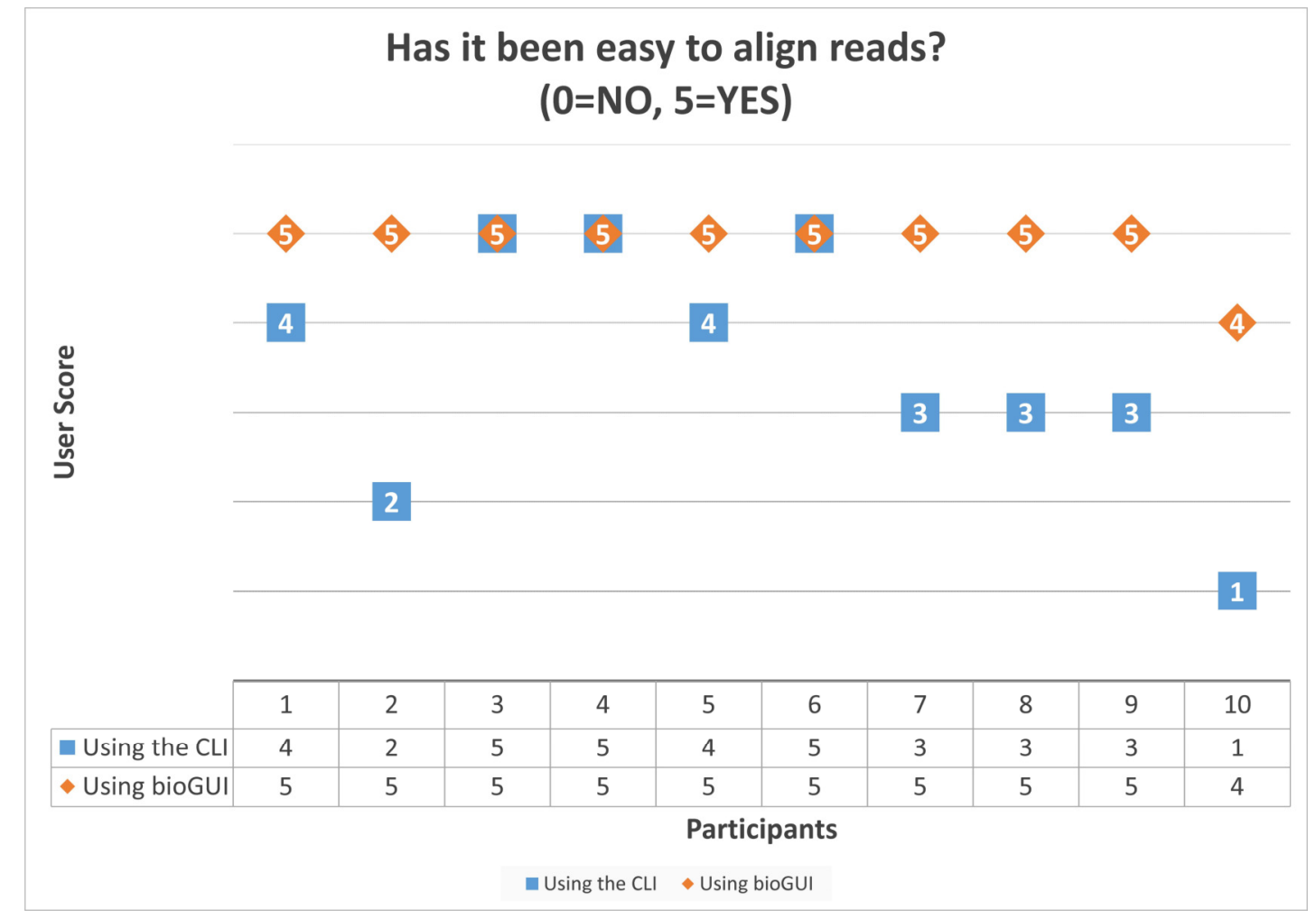

Figure A6. Scores given by the 10 participants on the question "Has it been easy to align the reads?" after performing the task using the CLI and bioGUI. These results show that most participants found the task easier using bioGUI, but for no-one it was harder to use bioGUI. 


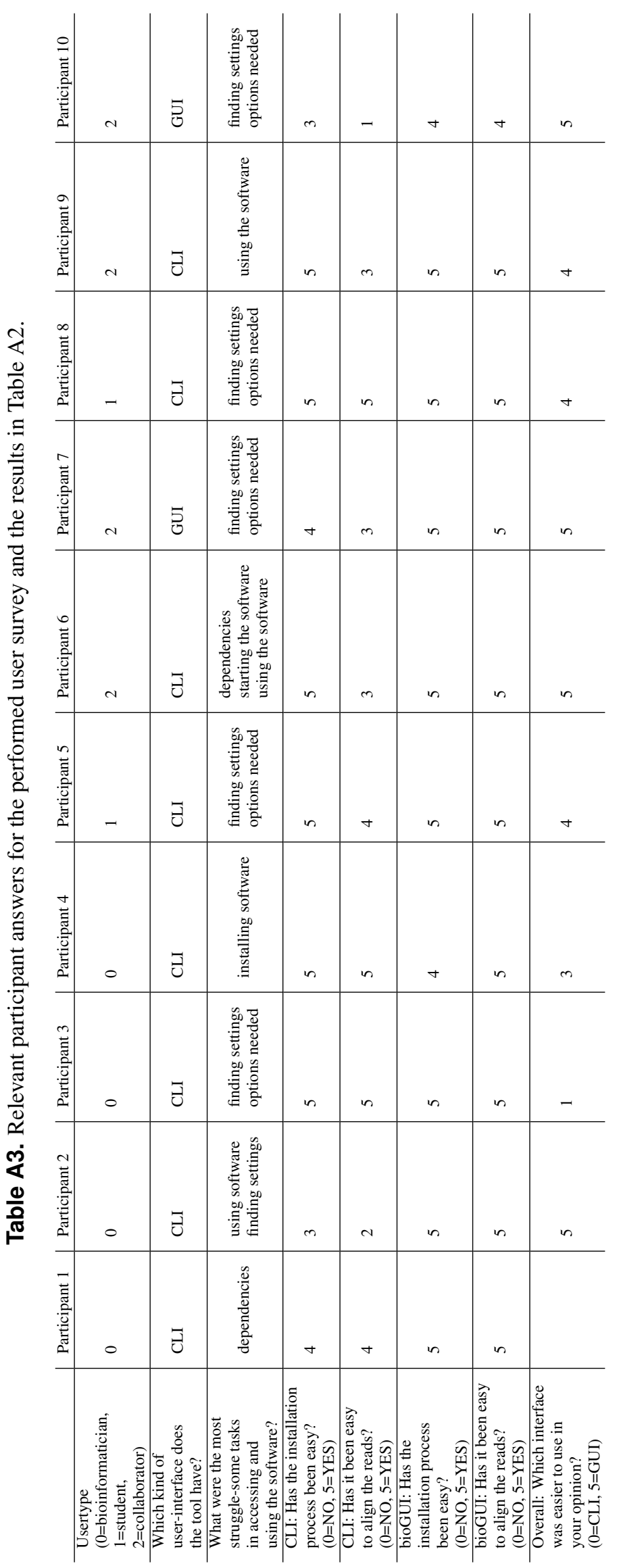

